EUROPEAN ORGANIZATION FOR NUCLEAR RESEARCH

CERN-EP/2003-087

10 December 2003

\title{
Search for fermiophobic Higgs bosons in final states with photons at LEP 2
}

DELPHI Collaboration

\begin{abstract}
Higgs boson production with subsequent decay to photons was searched for in the data collected by the DELPHI detector at centre-of-mass energies between $183 \mathrm{GeV}$ and $209 \mathrm{GeV}$, corresponding to a total integrated luminosity of nearly $650 \mathrm{pb}^{-1}$. No evidence for a signal was found, and limits were set on $h^{0} Z^{0}$ and $h^{0} A^{0}$ production with $h^{0}$ decay to photons. These results were used to exclude regions in the parameter space of fermiophobic scenarios of Two Higgs Doublet Models.
\end{abstract}

(Accepted by Euro. Phys. J. C) 


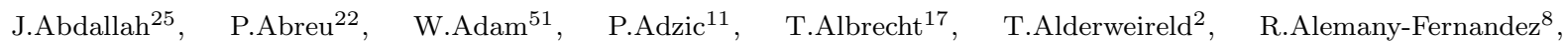
T.Allmendinger ${ }^{17}$, P.P.Allport ${ }^{23}$, U.Amaldi ${ }^{29}$, N.Amapane ${ }^{45}$, S.Amato ${ }^{48}$, E.Anashkin ${ }^{36}$, A.Andreazza ${ }^{28}$, S.Andringa ${ }^{22}$, N.Anjos ${ }^{22}$, P.Antilogus ${ }^{25}$, W-D.Apel ${ }^{17}$, Y.Arnoud ${ }^{14}$, S.Ask ${ }^{26}$, B.Asman ${ }^{44}$, J.E.Augustin ${ }^{25}$, A.Augustinus ${ }^{8}$, P.Baillon ${ }^{8}$, A.Ballestrero ${ }^{46}$, P.Bambade ${ }^{20}$, R.Barbier ${ }^{27}$, D.Bardin ${ }^{16}$, G.J.Barker ${ }^{17}$, A.Baroncelli ${ }^{39}$, M.Battaglia ${ }^{8}$, M.Baubillier ${ }^{25}$, K-H.Becks ${ }^{53}$, M.Begalli ${ }^{6}$, A.Behrmann ${ }^{53}$, E.Ben-Haim ${ }^{20}$, N.Benekos ${ }^{32}$, A.Benvenuti ${ }^{5}$, C.Berat ${ }^{14}$, M.Berggren ${ }^{25}$, L.Berntzon $^{44}$, D.Bertrand ${ }^{2}$, M.Besancon ${ }^{40}$, N.Besson ${ }^{40}$, D.Bloch ${ }^{9}$, M.Blom ${ }^{31}$, M.Bluj ${ }^{52}$, M.Bonesini ${ }^{29}$, M.Boonekamp ${ }^{40}$, P.S.L.Booth ${ }^{23}$, G.Borisov ${ }^{21}$, O.Botner ${ }^{49}$, B.Bouquet ${ }^{20}$, T.J.V.Bowcock ${ }^{23}$, I.Boyko ${ }^{16}$, M.Bracko $^{43}$, R.Brenner ${ }^{49}$, E.Brodet ${ }^{35}$, P.Bruckman ${ }^{18}$, J.M.Brunet ${ }^{7}$, L.Bugge ${ }^{33}$, P.Buschmann ${ }^{53}$, M.Calvi $^{29}$, T.Camporesi ${ }^{8}$, V.Canale ${ }^{38}$, F.Carena $^{8}$, N.Castro ${ }^{22}$, F.Cavallo ${ }^{5}$ M.Chapkin ${ }^{42}$, Ph.Charpentier ${ }^{8}$, P.Checchia ${ }^{36}$, R.Chierici ${ }^{8}$, P.Chliapnikov ${ }^{42}$, J.Chudoba ${ }^{8}$, S.U.Chung ${ }^{8}$, K.Cieslik ${ }^{18}$, P.Collins ${ }^{8}$, R.Contri ${ }^{13}$, G.Cosme ${ }^{20}$, F.Cossutti ${ }^{47}$, M.J.Costa ${ }^{50}$, D.Crennell ${ }^{37}$, J.Cuevas ${ }^{34}$, J.D'Hondt $^{2}$, J.Dalmau ${ }^{44}$, T.da Silva ${ }^{48}$, W.Da Silva ${ }^{25}$, G.Della Ricca ${ }^{47}$, A.De Angelis ${ }^{47}$, W.De Boer ${ }^{17}$, C.De Clercq ${ }^{2}$, B.De Lotto ${ }^{47}$, N.De Maria ${ }^{45}$, A.De Min ${ }^{36}$, L.de Paula ${ }^{48}$, L.Di Ciaccio ${ }^{38}$, A.Di Simone ${ }^{39}$, K.Doroba ${ }^{52}$, J.Drees ${ }^{53,8}$, M.Dris $^{32}$, G.Eigen ${ }^{4}$, T.Ekelof ${ }^{49}$, M.Ellert ${ }^{49}$, M.Elsing ${ }^{8}$, M.C.Espirito Santo ${ }^{22}$, G.Fanourakis ${ }^{11}$, D.Fassouliotis ${ }^{11,3}$, M.Feindt ${ }^{17}$, J.Fernandez ${ }^{41}$, A.Ferrer ${ }^{50}$, F.Ferro ${ }^{13}$, U.Flagmeyer ${ }^{53}$, H.Foeth ${ }^{8}$, E.Fokitis ${ }^{32}$, F.Fulda-Quenzer ${ }^{20}$, J.Fuster ${ }^{50}$, M.Gandelman ${ }^{48}$, C.Garcia ${ }^{50}$, Ph.Gavillet ${ }^{8}$, E.Gazis ${ }^{32}$, R.Gokieli ${ }^{8,52}$, B.Golob ${ }^{43}$, G.Gomez-Ceballos ${ }^{41}$, P.Goncalves ${ }^{22}$, E.Graziani ${ }^{39}$, G.Grosdidier ${ }^{20}$, K.Grzelak ${ }^{52}$, J.Guy ${ }^{37}$, C.Haag ${ }^{17}$, A.Hallgren ${ }^{49}$, K.Hamacher ${ }^{53}$, K.Hamilton ${ }^{35}$, S.Haug ${ }^{33}$, F.Hauler ${ }^{17}$, V.Hedberg ${ }^{26}$, M.Hennecke ${ }^{17}$, H.Herr ${ }^{8}$, J.Hoffman ${ }^{52}$, S-O.Holmgren ${ }^{44}$, P.J.Holt ${ }^{8}$, M.A.Houlden ${ }^{23}$, K.Hultqvist ${ }^{44}$, J.N.Jackson ${ }^{23}$, G.Jarlskog ${ }^{26}$, P.Jarry ${ }^{40}$, D.Jeans ${ }^{35}$, E.K.Johansson ${ }^{44}$, P.D.Johansson ${ }^{44}$, P.Jonsson ${ }^{27}$, C.Joram ${ }^{8}$, L.Jungermann ${ }^{17}$, F.Kapusta ${ }^{25}$, S.Katsanevas ${ }^{27}$, E.Katsoufis ${ }^{32}$, G.Kernel ${ }^{43}$, B.P.Kersevan ${ }^{8,43}$, U.Kerzel ${ }^{17}$, A.Kiiskinen ${ }^{15}$, B.T.King ${ }^{23}$, N.J.Kjaer ${ }^{8}$, P.Kluit ${ }^{31}$, P.Kokkinias ${ }^{11}$, C.Kourkoumelis ${ }^{3}$, O.Kouznetsov ${ }^{16}$, Z.Krumstein ${ }^{16}$, M.Kucharczyk ${ }^{18}$, J.Lamsa ${ }^{1}$, G.Leder ${ }^{51}$, F.Ledroit ${ }^{14}$, L.Leinonen ${ }^{44}$, R.Leitner ${ }^{30}$, J.Lemonne ${ }^{2}$, V.Lepeltier ${ }^{20}$, T.Lesiak ${ }^{18}$, W.Liebig ${ }^{53}$, D.Liko ${ }^{51}$, A.Lipniacka ${ }^{44}$, J.H.Lopes ${ }^{48}$, J.M.Lopez ${ }^{34}$, D.Loukas ${ }^{11}$, P.Lutz ${ }^{40}$, L.Lyons ${ }^{35}$, J.MacNaughton ${ }^{51}$, A.Malek ${ }^{53}$, S.Maltezos ${ }^{32}$, F.Mandl ${ }^{51}$, J.Marco ${ }^{41}$, R.Marco ${ }^{41}$, B.Marechal ${ }^{48}$, M.Margoni ${ }^{36}$, J-C.Marin ${ }^{8}$, C.Mariotti ${ }^{8}$, A.Markou ${ }^{11}$, C.Martinez-Rivero ${ }^{41}$, J.Masik ${ }^{12}$, N.Mastroyiannopoulos ${ }^{11}$, F.Matorras ${ }^{41}$, C.Matteuzzi ${ }^{29}$, F.Mazzucato ${ }^{36}$, M.Mazzucato $^{36}$, R.Mc Nulty ${ }^{23}$, C.Meroni ${ }^{28}$, E.Migliore ${ }^{45}$, W.Mitaroff ${ }^{51}$, U.Mjoernmark ${ }^{26}$, T.Moa ${ }^{44}$, M.Moch ${ }^{17}$, K.Moenig ${ }^{8,10}$, R.Monge ${ }^{13}$, J.Montenegro ${ }^{31}$, D.Moraes ${ }^{48}$, S.Moreno ${ }^{22}$, P.Morettini ${ }^{13}$, U.Mueller ${ }^{53}$, K.Muenich ${ }^{53}$, M.Mulders ${ }^{31}$, L.Mundim ${ }^{6}$, W.Murray ${ }^{37}$, B.Muryn ${ }^{19}$, G.Myatt ${ }^{35}$, T.Myklebust ${ }^{33}$, M.Nassiakou ${ }^{11}$, F.Navarria ${ }^{5}$, K.Nawrocki ${ }^{52}$, R.Nicolaidou ${ }^{40}$, M.Nikolenko ${ }^{16,9}$, A.Oblakowska-Mucha ${ }^{19}$, V.Obraztsov ${ }^{42}$, A.Olshevski ${ }^{16}$, A.Onofre ${ }^{22}$, R.Orava ${ }^{15}$, K.Osterberg ${ }^{15}$, A.Ouraou ${ }^{40}$, A.Oyanguren ${ }^{50}$, M.Paganoni ${ }^{29}$, S.Paiano ${ }^{5}$, J.P.Palacios ${ }^{23}$, H.Palka ${ }^{18}$, Th.D.Papadopoulou ${ }^{32}$, L.Pape ${ }^{8}$, C.Parkes ${ }^{24}$, F.Parodi ${ }^{13}$, U.Parzefall ${ }^{8}$, A.Passeri ${ }^{39}$, O.Passon ${ }^{53}$, L.Peralta ${ }^{22}$, V.Perepelitsa ${ }^{50}$, A.Perrotta ${ }^{5}$, A.Petrolini ${ }^{13}$, J.Piedra ${ }^{41}$, L.Pieri ${ }^{39}$, F.Pierre ${ }^{40}$, M.Pimenta ${ }^{22}$, E.Piotto ${ }^{8}$, T.Podobnik ${ }^{43}$, V.Poireau ${ }^{8}$, M.E.Pol ${ }^{6}$, G.Polok ${ }^{18}$, V.Pozdniakov ${ }^{16}$, N.Pukhaeva ${ }^{2,16}$, A.Pullia ${ }^{29}$, J.Rames ${ }^{12}$, A.Read ${ }^{33}$, P.Rebecchi ${ }^{8}$, J.Rehn ${ }^{17}$, D.Reid ${ }^{31}$, R.Reinhardt ${ }^{53}$, P.Renton ${ }^{35}$, F.Richard ${ }^{20}$, J.Ridky ${ }^{12}$, M.Rivero ${ }^{41}$, D.Rodriguez ${ }^{41}$, A.Romero ${ }^{45}$, P.Ronchese $^{36}$, P.Roudeau ${ }^{20}$, T.Rovelli ${ }^{5}$, V.Ruhlmann-Kleider ${ }^{40}$, D.Ryabtchikov ${ }^{42}$, A.Sadovsky ${ }^{16}$, L.Salmi ${ }^{15}$, J.Salt ${ }^{50}$, C.Sander ${ }^{17}$, A.Savoy-Navarro ${ }^{25}$, U.Schwickerath ${ }^{8}$, A.Segar ${ }^{35}$, R.Sekulin ${ }^{37}$, M.Siebel ${ }^{53}$, A.Sisakian ${ }^{16}$, G.Smadja ${ }^{27}$, O.Smirnova ${ }^{26}$, A.Sokolov ${ }^{42}$, A.Sopczak ${ }^{21}$, R.Sosnowski ${ }^{52}$, T.Spassov ${ }^{8}$, M.Stanitzki ${ }^{17}$, A.Stocchi ${ }^{20}$, J.Strauss ${ }^{51}$, B.Stugu ${ }^{4}$, M.Szczekowski ${ }^{52}$, M.Szeptycka ${ }^{52}$, T.Szumlak ${ }^{19}$, T.Tabarelli ${ }^{29}$, A.C.Taffard ${ }^{23}$, F.Tegenfeldt ${ }^{49}$, J.Timmermans ${ }^{31}$, L.Tkatchev ${ }^{16}$, M.Tobin ${ }^{23}$, S.Todorovova ${ }^{12}$, B.Tome ${ }^{22}$, A.Tonazzo ${ }^{29}$, P.Tortosa ${ }^{50}$, P.Travnicek ${ }^{12}$, D.Treille ${ }^{8}$, G.Tristram ${ }^{7}$, M.Trochimczuk ${ }^{52}$, C.Troncon ${ }^{28}$, M-L.Turluer ${ }^{40}$, I.A.Tyapkin ${ }^{16}$, P.Tyapkin ${ }^{16}$, S.Tzamarias ${ }^{11}$, V.Uvarov ${ }^{42}$, G.Valenti ${ }^{5}$, P.Van Dam ${ }^{31}$, J.Van Eldik ${ }^{8}$, A.Van Lysebetten ${ }^{2}$, N.van Remortel ${ }^{2}$, I.Van Vulpen ${ }^{8}$, G.Vegni ${ }^{28}$, F.Veloso ${ }^{22}$, W.Venus ${ }^{37}$, P.Verdier ${ }^{27}$, V.Verzi ${ }^{38}$, D.Vilanova ${ }^{40}$, L.Vitale ${ }^{47}$, V.Vrba $^{12}$, H.Wahlen ${ }^{53}$, A.J.Washbrook ${ }^{23}$, C.Weiser ${ }^{17}$, D.Wicke ${ }^{8}$, J.Wickens $^{2}$, G.Wilkinson ${ }^{35}$, M.Winter ${ }^{9}$, M.Witek ${ }^{18}$, O.Yushchenko ${ }^{42}$, A.Zalewska ${ }^{18}$, P.Zalewski ${ }^{52}$, D.Zavrtanik ${ }^{43}$, V.Zhuravlov ${ }^{16}$, N.I.Zimin ${ }^{16}$, A.Zintchenko ${ }^{16}$, M.Zupan ${ }^{11}$ 
${ }^{1}$ Department of Physics and Astronomy, Iowa State University, Ames IA 50011-3160, USA

${ }^{2}$ Physics Department, Universiteit Antwerpen, Universiteitsplein 1, B-2610 Antwerpen, Belgium and IIHE, ULB-VUB, Pleinlaan 2, B-1050 Brussels, Belgium

and Faculté des Sciences, Univ. de l'Etat Mons, Av. Maistriau 19, B-7000 Mons, Belgium

${ }^{3}$ Physics Laboratory, University of Athens, Solonos Str. 104, GR-10680 Athens, Greece

${ }^{4}$ Department of Physics, University of Bergen, Allégaten 55, NO-5007 Bergen, Norway

${ }^{5}$ Dipartimento di Fisica, Università di Bologna and INFN, Via Irnerio 46, IT-40126 Bologna, Italy

${ }^{6}$ Centro Brasileiro de Pesquisas Físicas, rua Xavier Sigaud 150, BR-22290 Rio de Janeiro, Brazil

and Depto. de Física, Pont. Univ. Católica, C.P. 38071 BR-22453 Rio de Janeiro, Brazil

and Inst. de Física, Univ. Estadual do Rio de Janeiro, rua São Francisco Xavier 524, Rio de Janeiro, Brazil

${ }^{7}$ Collège de France, Lab. de Physique Corpusculaire, IN2P3-CNRS, FR-75231 Paris Cedex 05, France

${ }^{8}$ CERN, CH-1211 Geneva 23, Switzerland

${ }^{9}$ Institut de Recherches Subatomiques, IN2P3 - CNRS/ULP - BP20, FR-67037 Strasbourg Cedex, France

${ }^{10}$ Now at DESY-Zeuthen, Platanenallee 6, D-15735 Zeuthen, Germany

${ }^{11}$ Institute of Nuclear Physics, N.C.S.R. Demokritos, P.O. Box 60228, GR-15310 Athens, Greece

${ }_{12}$ FZU, Inst. of Phys. of the C.A.S. High Energy Physics Division, Na Slovance 2, CZ-180 40, Praha 8, Czech Republic

${ }^{13}$ Dipartimento di Fisica, Università di Genova and INFN, Via Dodecaneso 33, IT-16146 Genova, Italy

${ }^{14}$ Institut des Sciences Nucléaires, IN2P3-CNRS, Université de Grenoble 1, FR-38026 Grenoble Cedex, France

${ }^{15}$ Helsinki Institute of Physics, P.O. Box 64, FIN-00014 University of Helsinki, Finland

${ }^{16}$ Joint Institute for Nuclear Research, Dubna, Head Post Office, P.O. Box 79, RU-101 000 Moscow, Russian Federation

${ }^{17}$ Institut für Experimentelle Kernphysik, Universität Karlsruhe, Postfach 6980, DE-76128 Karlsruhe, Germany

${ }^{18}$ Institute of Nuclear Physics PAN,Ul. Radzikowskiego 152, PL-31142 Krakow, Poland

${ }^{19}$ Faculty of Physics and Nuclear Techniques, University of Mining and Metallurgy, PL-30055 Krakow, Poland

${ }^{20}$ Université de Paris-Sud, Lab. de l'Accélérateur Linéaire, IN2P3-CNRS, Bât. 200, FR-91405 Orsay Cedex, France

${ }^{21}$ School of Physics and Chemistry, University of Lancaster, Lancaster LA1 4YB, UK

${ }^{22}$ LIP, IST, FCUL - Av. Elias Garcia, 14-1 ${ }^{\circ}$, PT-1000 Lisboa Codex, Portugal

${ }^{23}$ Department of Physics, University of Liverpool, P.O. Box 147, Liverpool L69 3BX, UK

${ }^{24}$ Dept. of Physics and Astronomy, Kelvin Building, University of Glasgow, Glasgow G12 8QQ

${ }^{25}$ LPNHE, IN2P3-CNRS, Univ. Paris VI et VII, Tour 33 (RdC), 4 place Jussieu, FR-75252 Paris Cedex 05, France

${ }^{26}$ Department of Physics, University of Lund, Sölvegatan 14, SE-223 63 Lund, Sweden

${ }^{27}$ Université Claude Bernard de Lyon, IPNL, IN2P3-CNRS, FR-69622 Villeurbanne Cedex, France

${ }^{28}$ Dipartimento di Fisica, Università di Milano and INFN-MILANO, Via Celoria 16, IT-20133 Milan, Italy

${ }^{29}$ Dipartimento di Fisica, Univ. di Milano-Bicocca and INFN-MILANO, Piazza della Scienza 2, IT-20126 Milan, Italy

${ }^{30}$ IPNP of MFF, Charles Univ., Areal MFF, V Holesovickach 2, CZ-180 00, Praha 8, Czech Republic

${ }^{31}$ NIKHEF, Postbus 41882, NL-1009 DB Amsterdam, The Netherlands

${ }^{32}$ National Technical University, Physics Department, Zografou Campus, GR-15773 Athens, Greece

${ }^{33}$ Physics Department, University of Oslo, Blindern, NO-0316 Oslo, Norway

${ }^{34}$ Dpto. Fisica, Univ. Oviedo, Avda. Calvo Sotelo s/n, ES-33007 Oviedo, Spain

${ }^{35}$ Department of Physics, University of Oxford, Keble Road, Oxford OX1 3RH, UK

${ }^{36}$ Dipartimento di Fisica, Università di Padova and INFN, Via Marzolo 8, IT-35131 Padua, Italy

${ }^{37}$ Rutherford Appleton Laboratory, Chilton, Didcot OX11 OQX, UK

${ }^{38}$ Dipartimento di Fisica, Università di Roma II and INFN, Tor Vergata, IT-00173 Rome, Italy

${ }^{39}$ Dipartimento di Fisica, Università di Roma III and INFN, Via della Vasca Navale 84, IT-00146 Rome, Italy

${ }^{40}$ DAPNIA/Service de Physique des Particules, CEA-Saclay, FR-91191 Gif-sur-Yvette Cedex, France

${ }^{41}$ Instituto de Fisica de Cantabria (CSIC-UC), Avda. los Castros s/n, ES-39006 Santander, Spain

${ }^{42}$ Inst. for High Energy Physics, Serpukov P.O. Box 35, Protvino, (Moscow Region), Russian Federation

${ }^{43}$ J. Stefan Institute, Jamova 39, SI-1000 Ljubljana, Slovenia and Laboratory for Astroparticle Physics,

Nova Gorica Polytechnic, Kostanjeviska 16a, SI-5000 Nova Gorica, Slovenia,

and Department of Physics, University of Ljubljana, SI-1000 Ljubljana, Slovenia

${ }^{44}$ Fysikum, Stockholm University, Box 6730, SE-113 85 Stockholm, Sweden

${ }^{45}$ Dipartimento di Fisica Sperimentale, Università di Torino and INFN, Via P. Giuria 1, IT-10125 Turin, Italy

${ }^{46}$ INFN,Sezione di Torino, and Dipartimento di Fisica Teorica, Università di Torino, Via P. Giuria 1, IT-10125 Turin, Italy

${ }^{47}$ Dipartimento di Fisica, Università di Trieste and INFN, Via A. Valerio 2, IT-34127 Trieste, Italy and Istituto di Fisica, Università di Udine, IT-33100 Udine, Italy

${ }^{48}$ Univ. Federal do Rio de Janeiro, C.P. 68528 Cidade Univ., Ilha do Fundão BR-21945-970 Rio de Janeiro, Brazil

${ }^{49}$ Department of Radiation Sciences, University of Uppsala, P.O. Box 535, SE-751 21 Uppsala, Sweden

${ }^{50}$ IFIC, Valencia-CSIC, and D.F.A.M.N., U. de Valencia, Avda. Dr. Moliner 50, ES-46100 Burjassot (Valencia), Spain

${ }^{51}$ Institut für Hochenergiephysik, Österr. Akad. d. Wissensch., Nikolsdorfergasse 18, AT-1050 Vienna, Austria

${ }^{52}$ Inst. Nuclear Studies and University of Warsaw, Ul. Hoza 69, PL-00681 Warsaw, Poland

${ }^{53}$ Fachbereich Physik, University of Wuppertal, Postfach 100 127, DE-42097 Wuppertal, Germany 


\section{Introduction}

In the Standard Model (SM), the decay of the Higgs boson to photons is mediated by heavy charged particles (namely $W^{ \pm}$bosons and top-quarks). The corresponding branching ratio $B R\left(h^{0} \rightarrow \gamma \gamma\right)$ is below $0.1 \%$. At LEP, this search is thus motivated by extensions of the SM. Many of the proposed models may enhance the $B R\left(h^{0} \rightarrow \gamma \gamma\right)$, either by enlarging the $h^{0} \gamma \gamma$ coupling [1] or by reducing the coupling of the Higgs boson to fermions [2].

We investigate the fermiophobic scenario of Two Higgs Doublets Models (2HDM) [2], in which the lightest CP-even Higgs boson decays to photons and can be produced together with a $Z^{0}$ or a CP-odd Higgs boson, $A^{0}$. The Higgs decay modes analysed in this paper are $h^{0} \rightarrow \gamma \gamma$ and $A^{0} \rightarrow b \bar{b}$ or $A^{0} \rightarrow h^{0} Z^{0}$. However, we consider also results for $h^{0} \rightarrow A^{0} A^{0}$ and for long-lived $A^{0}$, as described in [3]. All LEP2 data with centre-of-mass energies above $180 \mathrm{GeV}$ are analysed and the results reported here update those in [3].

The results on $h^{0} Z^{0}$ production with $h^{0} \rightarrow \gamma \gamma$ have been interpreted in several frameworks: previous analyses of DELPHI data can be found in [4] and results from the other LEP experiments can be found in [5]. In all cases, $h^{0} Z^{0}$ production with subsequent $h^{0} \rightarrow \gamma \gamma$ decay has been used as a benchmark in the search for particles with SM Higgslike couplings to bosons but no coupling to fermions.

In sections 2 and 3 , the $2 \mathrm{HDM}$ fermiophobic scenario and the data samples used will be introduced; the general selection of events with isolated photons will then be presented (in section 4), before going into the analysis dedicated to each of the $h^{0}$ boson production mechanisms and the different final states. The results obtained for $h^{0} Z^{0}$ production and $h^{0} A^{0}$ production (in sections 5 and 6 , respectively) are then combined to exclude regions in the parameter space of the 2HDM fermiophobic scenario, in section 7 .

\section{The 2HDM fermiophobic scenario}

General Two Higgs Doublets Models without explicit CP violation are characterized by five physical Higgs bosons: two neutral CP-even bosons $\left(h^{0}\right.$ and $\left.H^{0}\right)$, two charged bosons $\left(H^{ \pm}\right)$and one neutral CP-odd boson $\left(A^{0}\right)$. Together with the masses, the important parameters describing 2HDMs are the mixing angle in the neutral CP-even sector $(\alpha)$ and the ratio of the vacuum expectation values of the two Higgs doublets $(\tan \beta)$.

The couplings of the Higgs doublets to fermions could be realized in different ways, one possibility is that only one of the doublets couples to fermions. The coupling of the lightest CP-even boson to a fermion pair is then proportional to $\cos \alpha$. If $\alpha=\pi / 2$, this coupling vanishes and $h^{0}$ becomes a fermiophobic Higgs [2]: it decays to pairs of other Higgs bosons or massive gauge bosons when kinematically allowed, or to two photons in a large region of the parameter space.

In 2HDMs, the main mechanisms for the production of neutral Higgs bosons at LEP are $e^{+} e^{-} \rightarrow h^{0} Z^{0}$ and $e^{+} e^{-} \rightarrow h^{0} A^{0}$, both proceeding via $Z^{0}$ exchange. The two processes have complementary cross-sections proportional to $\sin ^{2} \delta$ and $\cos ^{2} \delta$, respectively, where $\delta=\alpha-\beta$. The $\sin \delta$ factor rescales the $h^{0} Z^{0} Z^{0}$ vertex with respect to the SM one.

It must be noticed that there are two different Higgs potentials which conserve CP [2] (referred to as potential A and B), each of them a function of seven parameters - the masses of the Higgs bosons, the $\alpha$ and $\beta$ angles and the sum of the squares of the vacuum expectation values for the two doublets. The choice of the potential does not affect the Higgs interactions with gauge bosons or fermions but leads to different Higgs-Higgs 
couplings and thus different phenomenologies. In particular, the decay width of $h^{0} \rightarrow \gamma \gamma$, to which diagrams with $H^{ \pm}$loops significantly contribute, can be changed.

For potential A, the branching ratio of the lightest CP-even Higgs to two photons $\mathrm{BR}\left(h^{0} \rightarrow \gamma \gamma\right)$ depends on $M_{h^{0}}$, and weakly on $\sin ^{2} \delta$. Under the assumption that $M_{H^{0}} \sim 1 \mathrm{TeV} / c^{2}$ and that $h^{0}$ does not decay to other Higgs particles, the $\operatorname{BR}\left(h^{0} \rightarrow \gamma \gamma\right)$ for $\sin ^{2} \delta=1$ can be obtained using the SM couplings to bosons and no coupling to fermions. For other values of $\sin ^{2} \delta$, it increases with respect to this benchmark model. For potential $\mathrm{B}$, the $\mathrm{BR}\left(h^{0} \rightarrow \gamma \gamma\right)$ depends also on $M_{A^{0}}$ and $M_{H^{ \pm}}$and there can be large cancellations between the several loop contributions for some values of these parameters. In both cases, $h^{0} \rightarrow A^{0} A^{0}$ is dominant when $M_{h^{0}}>2 M_{A^{0}}$.

The widths for the $A^{0}$ tree level decays (to $f \bar{f}, Z^{0} h^{0}$ and $W^{ \pm} H^{\mp}$ ) are independent of the potential but depend on $M_{A^{0}}, M_{h^{0}}$ and $\sin ^{2} \delta$. For low values of $\sin ^{2} \delta$, also $A^{0}$ becomes fermiophobic and light $A^{0}$ bosons can thus be long lived.

The low values of $\sin ^{2} \delta$ lead also to different mass configurations, according to the choice of potential. For potential A they imply $M_{h^{0}} \sim 0$ and for potential B $M_{h^{0}} \sim M_{A^{0}}$.

\section{Data samples}

The data analysed were taken by the DELPHI detector at LEP in the years from 1997 to 2000. The corresponding average centre-of-mass energies $(\sqrt{s})$ and integrated luminosities $(\mathcal{L})$ are shown in Table 1.

\begin{tabular}{|c||r|r|r|r|r|r|r|r|r|}
\hline year & 1997 & 1998 & \multicolumn{4}{|c|}{1999} & \multicolumn{3}{|c|}{2000} \\
\hline$\sqrt{s}(\mathrm{GeV})$ & 182.6 & 188.6 & 191.6 & 195.5 & 199.6 & 201.6 & 205.0 & 206.5 & 206.8 \\
$\mathcal{L}\left(\mathrm{pb}^{-1}\right)$ & 49.3 & 153.0 & 25.1 & 76.0 & 82.7 & 40.2 & 80.0 & 59.2 & 81.8 \\
\hline
\end{tabular}

Table 1: Average centre-of-mass energies and integrated luminosities of the analysed samples. The sample with $\sqrt{s}=206.5 \mathrm{GeV}$ corresponds to the data collected after the damage on the TPC.

In the year 2000, the centre-of-mass energies ranged from $200 \mathrm{GeV}$ to $209 \mathrm{GeV}$, while most of the luminosity was collected at around $205 \mathrm{GeV}$ and $207 \mathrm{GeV}$. In the last part of the running, DELPHI suffered irreparable damage to one of the sectors of the main tracking device, the Time Projection Chamber (TPC), representing 1/12 of the acceptance. These data were analysed separately, to isolate any systematic difference.

The DELPHI apparatus and performance are described in detail in [6,7]. The tracking system of DELPHI consisted of the TPC and a Vertex Detector (VD) closest to the beam pipe and was supplemented by extra tracking detectors, the Inner and Outer Detectors in the barrel region, and two Forward Chambers. It was embedded in a magnetic field of 1.2 T, aligned parallel to the beam axis. The most important subdetectors for the present analysis were the electromagnetic calorimeters covering different polar angle regions ${ }^{1}$ : the luminosity monitor (STIC) for $\theta$ below $11^{\circ}$, the Forward ElectroMagnetic Calorimeter (FEMC) between $11^{\circ}$ and $35^{\circ}$ and the High density Projection Chamber (HPC) above $42^{\circ}$. The regions in between the FEMC and the HPC and the HPC intermodular division at $\theta=90^{\circ}$ were equipped with hermeticity counters - scintillators covered with lead, so that photons could also be tagged there. In the azimuthal intermodular divisions of the

\footnotetext{
${ }^{1}$ The polar angle, $\theta$, is defined in relation to the beam axis. In all cases the complementary value $\left(180^{\circ}-\theta\right)$ is also assumed. The azimuthal angle, $\phi$, is the angle in the plane perpendicular to the beam direction.
} 
$\mathrm{HPC}$, at $\bmod \left(\phi, 15^{\circ}\right)=7.5^{\circ}$, the detection of photons could be complemented by the use of the Hadronic CALorimeter (HCAL). The hadronic calorimeter covered $98 \%$ of the total solid angle, down to $11^{\circ}$, and the whole detector was surrounded by muon drift chambers. The major hardware change with respect to the description in [7] was the inclusion of the Very Forward Tracker [8] which extended the coverage of the Vertex Detector down to a polar angle of $11^{\circ}$. Together with new tracking algorithms, and new alignment and calibration procedures, this led to an improved track reconstruction efficiency in the forward regions of DELPHI. The tracking algorithms for the barrel part of DELPHI were also changed to recuperate efficiency in the damaged TPC sector.

Events corresponding to the SM processes and Higgs production signals were fully simulated for each data set. The main background processes were generated with KK2f [9] (for $e^{+} e^{-} \rightarrow q \bar{q}(\gamma)$ ), KoralZ [10] (for $e^{+} e^{-} \rightarrow \nu \bar{\nu}(\gamma)$ and $e^{+} e^{-} \rightarrow l^{+} l^{-}(\gamma)$ ) and Bhwide [11] (for the Bhabha scattering), all of which include a detailed description of the initial state radiation. These processes constitute irreducible sources of background, and thus their accurate description is a crucial point in the analysis. In a previous paper [3], the $q \bar{q}(\gamma)$ background had been simulated with Pythia 6.1 [12]; KK2f has a more accurate description of the initial state photon radiation and gives a 30\% increase in the crosssection for the production of $q \bar{q}$ with two photons at high polar angle. It also has a better match to the DELPHI data as shown in the next sections.

Other SM processes give smaller contributions to the studied channels. All the 4-fermion processes (including neutral and charged currents) were generated as described in [13], using the WPHACT generator [14], and complemented with samples of multiperipheral production of $e^{+} e^{-} f \bar{f}$ events generated with $\mathrm{BDK} / \mathrm{BDKRC}[15]$ in the regions dominated by virtual photon collisions. The QED $e^{+} e^{-} \rightarrow \gamma \gamma$ process and Compton events were generated with RADCOR [16] and TEEG [17], respectively. The hadronization and fragmentation in hadronic final states was performed by Pythia [12].

Signal processes corresponding to the different analysed final states were generated according to Pythia 6.1 [12] at the different average centre-of-mass energies, for different values of the Higgs bosons masses (from 3 to $120 \mathrm{GeV} / \mathrm{c}^{2}$ for the $h^{0}$ boson and 10 to $170 \mathrm{GeV} / \mathrm{c}^{2}$ fro the $A^{0}$ boson). They were cross-checked with a dedicated generator HZHA [18].

All the generated data sets at the different centre-of-mass energies were passed through the DELPHI simulation and the same reconstruction chain as data [7]. Dedicated samples of all the above processes were used to simulate the effect of the damaged TPC sector. Its impact on the analyses was found to be negligible within the statistical uncertainties.

\section{Selection of events with isolated photons}

The selection of events with isolated photons is common to all analyses and is described in this section. First a general selection was applied and then isolated photons and leptons were reconstructed.

Only events with visible energy in the polar angle region above $20^{\circ}$ greater than $0.1 \sqrt{s}$ were accepted. In addition, all events were required to contain at least one charged or neutral object with energy above $5 \mathrm{GeV}$ in the same polar angle region. This vetoed most of the contamination from virtual photon collision events.

Charged particles were classified as 'good' if they had measured momentum greater than $0.1 \mathrm{GeV} / c$ and their tracks were reconstructed with impact parameters to the interaction point below $4 \mathrm{~cm}$ in the transverse plane and $4 / \sin \theta \mathrm{cm}$ in the beam direction. 
Energy deposits in the calorimeters unassociated to charged particles were required to be above $0.3 \mathrm{GeV}$ to be classified as neutral particles.

The reconstruction of photons was done in several steps, starting from the showers in the electromagnetic calorimeters. The procedure described in [7] was followed to identify tight photon candidates in the HPC. This algorithm selects electromagnetic energy deposits with shower profiles compatible with those of photons. However, showers close to the HPC divisions were accepted as loose photon candidates even if they failed the transverse shower profile criteria (and/or the longitudinal shower profile criteria if their energy was above $25 \mathrm{GeV}$ ). In the forward region, all STIC energy deposits with polar angle satisfying $\theta<11^{\circ}$ were taken to be tight photon candidates ${ }^{2}$. An algorithm was used to correct for the effects of photon conversion and shower development in the detector material in front of the FEMC, as explained below. Electromagnetic deposits close in space were clustered together and the association with reconstructed charged particle tracks was used for electron/photon discrimination. Tight photon candidates were required to have no association to VD track elements, nor to signals from different combinations of other tracking detectors (depending on the shower polar angle). Care was taken to exclude those tracks which were likely to come from the development of showers outside the calorimeter. Loose photon candidates were allowed to have two associated tracks. In addition, the ratio of electromagnetic energy to the total energy around the cluster - in an angular region defined by $|\Delta \theta|<15^{\circ}$ and $|\Delta \phi|<\min \left(15^{\circ}, 6^{\circ} \cot \theta_{\text {cluster }}\right)-$ was required to be above $90 \%$.

The identification of isolated photons started from the candidates defined above and used a double cone centred around the photon axis, as explained below. Only isolated photons with energies above $5 \mathrm{GeV}$ were considered. The selection criteria depended on the topology of the event and were as follows.

For the topology with photons only, the total energy inside a cone with half-angle of $10^{\circ}$ was associated to the photon, while, to ensure isolation, the total energy between $10^{\circ}$ and $12^{\circ}$ was not permitted to exceed $5 \mathrm{GeV}$.

For the other topologies, the criteria were different for loose and tight photon candidates. For loose photon candidates, the half-angle of the inner cone was of $5^{\circ}$ and the energy between $5^{\circ}$ and $15^{\circ}$ was not permitted to exceed $1 \mathrm{GeV}$. In the case of photons tightly identified by the shower profile analysis, no further association was done and only the external cone was kept. The angle of the external cone, $\alpha$, was varied according to the energy of the photon candidate, down to $3^{\circ}$ for $E_{\gamma}>90 \mathrm{GeV}$. The energy limit inside the cone was rescaled to $\sin \alpha / \sin 15^{\circ} \mathrm{GeV}$, but one energetic particle was accepted inside this cone (and excluded from the energy calculation).

The identification of isolated photons in the barrel region required also that there was no HPC layer with more than $90 \%$ of their electromagnetic energy, while the hadronic energy depositions above $3 \mathrm{GeV}$ must be concentrated in the first layer of the HCAL.

The reconstruction of isolated leptons followed the same double cone criteria, starting from good charged particles with momentum above $4 \mathrm{GeV} / c$. The total charged particle momentum and energy deposition inside the inner cone around the charged particle track were associated, and an external cone used to ensure isolation. Isolated charged particles associated to signals in the muon chambers, and for which the ratio between the energy deposited in calorimeters and the measured momentum was less than $20 \%$, were identified as tight muon candidates. Electromagnetic showers associated to good charged particles, reconstructed with algorithms similar to those of photon reconstruction, were considered as tight or loose electron candidates.

\footnotetext{
${ }^{2}$ Energy depositions below $3^{\circ}$ were discarded from the events, to avoid contamination from off-momentum beam electrons.
} 
The separation between electrons and photons converting in the tracking system relied on the information from the VD and its association to isolated charged particles. A VD track element was defined as at least two signals in different layers of the detector, which were associated to an isolated particle if aligned within $3^{\circ}$ of its azimuthal direction $\left(10^{\circ}\right.$ in the forward region). Isolated charged particle tracks not associated to VD track elements were considered as candidates for photons converting in the tracking system. At most one converted photon candidate was allowed per event, except for the $l^{+} l^{-} \gamma \gamma$ topologies where no recovery of converted photons was performed.

\section{$5 \quad$ Search for $h^{0} Z^{0}$ production}

\subsection{Two photons and jets}

In $h^{0} Z^{0}$ production, the final state with highest branching fraction is the one corresponding to the $Z^{0}$ decay to hadrons, and $h^{0}$ decay to photons. The preselection of this topology required that at least six good charged particles were present and the visible energy in the polar angle region above $20^{\circ}$ was greater than $0.2 \sqrt{s}$. All selected charged and neutral particles, except for isolated photons and leptons, were clustered into two jets using the DURHAM jet algorithm [19]. Two isolated photons were required in the event, and extra isolated particles were allowed only if their transverse momentum with respect to one of the jets was less than $20 \mathrm{GeV} / c$.

The main irreducible background to this search was $q \bar{q}$ production with two photons coming predominantly from initial state radiation: at LEP2, about half of the $q \bar{q}$ events were radiative return events, with an effective collision energy around $M_{Z^{0}}$. Very small contributions from other sources, namely $W^{+} W^{-}$production, were also present.

After the selection of the two isolated photons in the event, further requirements on their isolation and polar angle reduced both Initial and Final State Radiation (ISR and FSR) contributions. The most energetic photon had to have at least $15 \%$ of the beam energy, the minimum transverse momentum of the photons with respect to the jets had to be above $7.5 \mathrm{GeV} / c$ and the minimum polar angle above $30^{\circ}$.

To improve the resolution on the invariant mass of the photon pair, a kinematic fit imposing energy-momentum conservation and constraining the jet-jet invariant mass to the $Z^{0}$ mass was performed according to [20]. The main inputs to the fit are the directions and energies of the photons, which are considerably less well measured if the photons are not well contained in the calorimeters. $\sigma_{E}$, the relative error on the measured energy $E_{\gamma i}^{\text {meas }}$, was obtained from calorimeter resolution studies. It was set to $100 \%$ for photons reconstructed in the calorimeter boundaries, and events in which both photons had $\sigma_{E}=100 \%$ were rejected. For the other events, a partial $\chi^{2}$ was constructed as:

$$
\frac{\chi_{\gamma \gamma}^{2}}{n d o f}=\frac{1}{2} \sum_{i=1,2}\left(\frac{E_{\gamma i}^{f i t}-E_{\gamma i}^{\text {meas }}}{\sigma_{E} E_{\gamma i}^{\text {meas }}}\right)^{2},
$$

If both photons were well contained in the calorimeters, it was required that $\chi_{\gamma \gamma}^{2} / n d o f$ was below 5. If one of the photons was not well contained, the information from the jets had a larger weight and it was the global $\chi^{2} / n d o f$ of the fit (defined in [20]) that was required to be below 5 .

At this stage, the contribution from non- $q \bar{q}(\gamma)$ events becomes almost negligible. The characteristics of the radiative return events were then used to reduce the background further. In most of these events, the photons are in the forward part of the detector and, 
usually, one of the photons carries most of the energy necessary to bring the $Z^{0}$ boson on-shell:

$$
E_{r e t}=\frac{s-M_{Z}^{2}}{2 \sqrt{s}}
$$

Only events in which the energy difference between the two photons was lower than $0.70 E_{\text {ret }}$ were kept. Figure 1 shows the sum and difference of the two photon energies normalized to the radiative return energy. In the final sample, one of the photons was required to be inside the HPC acceptance.

Table 2 shows, for the data collected in the year 2000, the evolution with the selection criteria of the number of selected data events, corresponding SM background and efficiencies for two Higgs signals of different masses. The contribution of the irreducible $q \bar{q}$ background is shown separately. The numbers of selected events in all data and simulated samples at the final selection level are shown in Table 5. The reconstructed jet-jet invariant masses and the fitted $\gamma \gamma$ masses are also shown in Figure 1.

\subsection{Two photons and two charged leptons}

The production of $h^{0} Z^{0}$ with subsequent decays $h^{0} \rightarrow \gamma \gamma$ and $Z^{0} \rightarrow l^{+} l^{-}$was analysed for all the three charged lepton flavours. A common preselection required the presence of two isolated photons and two isolated leptons in events with at most five good charged particles and visible energy in the polar angle region above $20^{\circ}$ greater than $0.2 \sqrt{s}$. The most energetic photon was required to have energy greater than $0.1 \sqrt{s}$ and the polar angle of the leptons was required to be above $20^{\circ}$ (except for the ones identified as muons).

The background processes for these channels include $e^{+} e^{-} \rightarrow Z^{0} / \gamma^{*} \rightarrow l^{+} l^{-}$and the $t$-channel Bhabha scattering, which has a very large cross-section. To reduce the number of events with FSR, the minimum transverse momentum of each photon with respect to any of the leptons was required to be greater than $5 \mathrm{GeV} / c$. The contribution from events with ISR, namely from radiative return to the $Z^{0}$, was reduced requiring that both photons had polar angles above $30^{\circ}$, that there was at least one photon in the HPC, and imposing that the difference between the energies of the two photons was below $0.7 E_{\text {ret }}$ (see equation 2).

A kinematic fit imposing energy-momentum conservation and using the measured directions of the four particles in the event was performed, to rescale the energies of leptons and photons. Partial $\chi^{2} / n d o f$ associated to the leptons or to the photons were defined, as in equation 1, for the photon energies and lepton momenta; events were accepted only if one of the two was below 10. Only events for which the fitted di-lepton invariant mass was between 60 and $120 \mathrm{GeV} / \mathrm{c}^{2}$ were kept.

Table 3 presents the number of selected events in the year 2000 data and the corresponding SM background expectations, at each selection level, together with the efficien-

cies for two Higgs masses. The selection efficiencies for the different leptonic decays of the $Z^{0}$ vary between $13 \%$ for $\tau^{+} \tau^{-}$and $38 \%$ for $\mu^{+} \mu^{-}$for a Higgs mass of $100 \mathrm{GeV} / c^{2}$. The final invariant mass spectra are shown in Figure 2, and the corresponding numbers of selected events in each data set are given in Table 5.

\subsection{Two photons and missing energy}

$h^{0} Z^{0}$ production with the $Z^{0}$ decay into neutrino pairs leads to purely photonic final states with large missing energy. All purely photonic candidate events were allowed to have at most 5 good charged particles, none of them associated to VD track elements or to 
signals in the muon chambers. Charged particles not associated to energy deposits above $5 \mathrm{GeV}$ had to have momenta below $5 \mathrm{GeV} / \mathrm{c}$ and the minimum transverse momentum of each individual particle with respect to the other particles reconstructed in the same hemisphere had to be greater than $5 \mathrm{GeV} / \mathrm{c}$.

Cosmic rays leaving energy deposits in the calorimeters are an important source of background for final states with photons and missing energy. Most cosmic ray events crossing the tracking system were removed by requiring all charged particles in the event to satisfy the impact parameter selection criteria defined in section 4. Cosmic ray events crossing the detector outside the tracking devices were vetoed by requiring that the total unassociated energy deposition in the event was less than $10 \%$ of the energy of the isolated photons. Events in which more than $98 \%$ of the energy of the photon candidates was reconstructed from depositions in the hadronic calorimeters were also vetoed. In addition, the direction of photons reconstructed in the HPC was required to be consistent within $25^{\circ}$ with the hypothesis that the particle was coming from the primary vertex.

After this preselection, only events with two photons reconstructed with polar angles above $25^{\circ}$ and well contained either in the FEMC or in the HPC were kept.

The main background process for this channel is the production of $\nu \bar{\nu}$ pairs (either through $Z^{0}$ or $W^{ \pm}$boson exchange) with the emission of ISR photons. Even though the cross-section is low, these events constitute an irreducible source of background. The background from the QED reaction $e^{+} e^{-} \rightarrow \gamma \gamma(\gamma)$, which has a large cross-section, was much reduced by appropriate kinematic requirements. Most of the QED events were vetoed by imposing that the angle between the two photons was lower than $178^{\circ}$ and that the energy of the most energetic photon was below $0.4 \sqrt{s}$. Additional criteria were applied to events with photons in the less efficient regions of the electromagnetic calorimeters. If one of the photons was either within $1.5^{\circ}$ of an azimuthal modular division of the HPC, or its polar angle corresponded to the HPC edges, (defined by the intervals $\left[42^{\circ}, 44^{\circ}\right]$ and $\left.\left[87^{\circ}, 88^{\circ}\right]\right)$, the acoplanarity ${ }^{3}$ was required to be above $3^{\circ}$ and $5^{\circ}$, respectively. If there was one converted photon in the FEMC, the acoplanarity was also required to be above $3^{\circ}$, in order to account for the larger deflection of charged particles in the forward region. Any signal observed in the hermeticity counters in between the FEMC and the HPC was required to be within $20^{\circ}$ of a reconstructed photon.

A kinematic fit was then performed imposing the $Z^{0}$ mass on the invisible system, after requiring that the missing mass of the selected event was larger than $20 \mathrm{GeV} / c^{2}$. The global $\chi^{2} / n d o f[20]$ resulting from the fit was required to be below 5 .

The results of the selection in the year 2000 data set are summarized in Table 4, where the SM expectations were corrected for trigger efficiencies ${ }^{4}$. The invariant mass spectra at the final selection level are shown in Figure 2, and the corresponding numbers of selected events in each data set are given in Table 5.

\subsection{Results on $h^{0} Z^{0}$ production}

A good agreement between data and SM expectations was found in all analysed channels, with a total of 54 events selected and $52 \pm 1$ expected. The numbers of selected events per channel and centre-of-mass energy are shown in Table 5.

Limits on the production cross-section of $h^{0} Z^{0}$ with $h^{0} \rightarrow \gamma \gamma$, as a function of $M_{h^{0}}$, were obtained by combining all the channels and centre-of-mass energies using the Mod-

\footnotetext{
${ }^{3}$ The acoplanarity between two objects is defined as the complement of the angle between them in the plane transverse to the beam direction.

${ }^{4}$ The trigger efficiencies for events with two photons in the barrel part of DELPHI were of $96 \%-98 \%$; in the forward part, as for other final states, they were of $\sim 100 \%$. In the other channels the effect of this correction is thus negligible.
} 
ified Frequentist Likelihood Ratio method [21], taking into account the measured and expected $M_{\gamma \gamma}$ invariant mass distributions. The charged lepton flavours were added in a single channel for each centre-of-mass energy.

The signal samples generated with Pythia 6.1 were cross-checked with samples generated with HZHA and found to be compatible within the statistical uncertainty of $1 \%$. Different fragmentation/hadronization models were also compared, and found to have negligible impact in the $q \bar{q} \gamma \gamma$ selection. Extra systematic effects could come from the parameterization of the expected invariant $\gamma \gamma$ mass distributions. They were found to be small when compared to the statistical uncertainty on the total background expectations. To account for all effects, a systematic error of $\pm 3 \%$ was assigned to the signal efficiency used in the limit calculation.

Figure 3 shows, as a function of the Higgs boson mass, the 95\% Confidence Level (CL) upper limit on $B R\left(h^{0} \rightarrow \gamma \gamma\right)$ times the ratio of the $h^{0} Z^{0}$ production cross-section to the $\mathrm{SM}$ one. This ratio is equal to $\sin ^{2} \delta$ in $2 \mathrm{HDM}$ (see section 2). For the range of masses studied, and taking the model in [2] as a reference, the width of the Higgs boson is always below the mass resolution of the analyses ${ }^{5}$.

In a model where the Higgs couplings to bosons have SM values, but the couplings to fermions vanish, a 95\% CL lower limit on the $h^{0}$ boson mass is given by the intersection of the cross-section limit and the prediction for $B R\left(h^{0} \rightarrow \gamma \gamma\right)$ (also shown in Figure 3), at $104.1 \mathrm{GeV} / c^{2}$. The expected limit in this case is $104.6 \mathrm{GeV} / c^{2}$.

\section{Search for $h^{0} A^{0}$ production}

\subsection{Two photons and two b-jets}

The search for $h^{0} A^{0} \rightarrow \gamma \gamma b \bar{b}$ is very similar to the one for $h^{0} Z^{0} \rightarrow \gamma \gamma q \bar{q}$ (see section 5.1). Similar criteria were applied, differing mainly in the kinematic fit performed. In the $h^{0} A^{0}$ case less constraints were used: the jet-jet invariant mass was left free and an additional ISR photon was allowed in the beam direction. In fact, if $M_{h^{0}}+M_{A^{0}}<M_{Z^{0}}$, a significant part of the signal events may correspond to radiative returns to the $Z^{0}$ with subsequent $Z^{0} \rightarrow h^{0} A^{0}$. Since $M_{A^{0}}$ has to be fitted without constraints, the global $\chi^{2} /$ ndof becomes an important selection criterion: it was required to be below 10 in all cases. Tighter requirements on partial $\chi_{\gamma \gamma}^{2} / n d o f$ and global $\chi^{2} / n d o f$ were still apllied as discussed for the $h^{0} Z^{0}$ case.

In the next stage, it was required that the energies of the two photons satisfied $\left|E_{\gamma 1}-E_{\gamma 2}\right|<0.70\left|E_{\gamma 1}+E_{\gamma 2}\right|$. Again, at least one of the photons had to be in the HPC and the combined b-tagging of the event, as defined in [22], was required to be above -2. Figure 4 shows the agreement of the b-tagging variable in data and SM simulation, together with the mass distributions obtained for the $b \bar{b}$ and $\gamma \gamma$ pairs.

The total numbers of selected data and background events in the analysis of the year 2000 data are shown in Table 6 . The non- $q \bar{q}$ background is larger compared to the case with the $Z^{0}$ mass constraint: it consists mainly of semi-leptonic $W^{+} W^{-}$events. Table 6 shows also the efficiency obtained for $e^{+} e^{-} \rightarrow h^{0} A^{0}$ with $M_{A^{0}}=M_{Z^{0}}$ and $M_{h^{0}}=100$ $\mathrm{GeV} / c^{2}$. The numbers of selected events at the final analysis level are given in Table 8 .

${ }^{5}$ For $M_{h^{0}}=100 \mathrm{GeV} / c^{2}$, the mass resolutions were of $1 \%$ for the leptonic channel, $2.5 \%$ in the hadronic channel, and $3.5 \%$ in the missing energy channel. 


\subsection{Three or more photons and jets}

Within the framework of general 2HDM, the main decay channel of $A^{0}$ is $b \bar{b}$. However, when kinematically allowed, the decay to $h^{0} Z^{0}$ becomes dominant in a large region of parameter space. This can lead to final states with a fermion pair and four photons. Only the $Z^{0}$ decay to quark pairs, which has the highest branching ratio, was analysed. At least three isolated photons were required and at most one converted photon was allowed.

Two of the photons had to be above $30^{\circ}$ in polar angle and have a transverse momentum with respect to any of the jets greater than $7.5 \mathrm{GeV} / \mathrm{c}$. The jet-jet invariant mass was required to be in the range 50 to $130 \mathrm{GeV} / c^{2}$. The difference between the energy of any of the photons and the energy carried by all the others had to be below $0.33 E_{\text {ret }}$ (defined in equation 2). Finally, one of the photons was required to be in the HPC, and at least three photons were required to be above $15^{\circ}$ in polar angle.

The numbers of selected events in the 2000 data sample and the corresponding SM expectations are given in Table 7 . The efficiencies for the signal were as high as $60 \%$ for high masses, and above $40 \%$ for most of the considered range. Since only three photons were required, no mass reconstruction was attempted and the result is based on the numbers of selected events at this stage (given in Table 8).

\subsection{Results on $h^{0} A^{0}$ production}

The numbers of selected events in each data set and for both channels are shown in Table 8. Limits were extracted with the same algorithm as for $h^{0} Z^{0}$. In the $b \bar{b} \gamma \gamma$ channel the reconstructed mass values were used, while for the $q \bar{q} \gamma \gamma \gamma(\gamma)$ there was no attempt to reconstruct the masses, and a pure counting experiment was performed. Searches in both channels had similar sensitivity to the signal.

To extract upper limits on $\cos ^{2} \delta$ (defined in section 2), reference cross-sections computed with HZHA [18] were used. For the $h^{0} \rightarrow \gamma \gamma$ decay, the branching ratio computed with $\sin ^{2} \delta=1$ and shown in Figure 3 was used. This represents a conservative assumption since $\operatorname{BR}\left(h^{0} \rightarrow \gamma \gamma\right)$ increases for lower $\sin ^{2} \delta$. Depending on the point in parameter space under consideration, the limit was derived assuming a $100 \%$ branching fraction for the dominant decay channel of the $A^{0}\left(b \bar{b}\right.$ or $\left.h^{0} Z^{0}\right)$. The limits are almost independent of the other model parameters, if the masses $M_{H^{0}}$ and $M_{H^{ \pm}}$are high enough not to open new decay channels as $A^{0} \rightarrow H^{ \pm} W^{\mp}$.

Figure 5 shows the limits on $\sin ^{2} \delta$ as a function of $M_{h^{0}}$, obtained for two different $M_{A^{0}}$ values - they strongly depend on the mass hypothesis used for $A^{0}$.

\section{Exclusion in the 2HDM parameter space}

The combination of the results on $h^{0} Z^{0}$ and $h^{0} A^{0}$ production is illustrated in Figure 5 . The upper limits on $\sin ^{2} \delta$ for a given $M_{h^{0}}$ and the upper limits on $\cos ^{2} \delta$ for a given $\left(M_{h^{0}}, M_{A^{0}}\right)$ pair are combined to exclude regions in the $\left(M_{h^{0}}, M_{A^{0}}\right)$ plane for all $\delta$ values.

Figure 6 shows the $95 \%$ CL exclusion in the plane $\left(M_{h^{0}}, M_{A^{0}}\right)$ divided into separate regions according to the different kinematics:

I. $M_{A^{0}}>M_{h^{0}}+M_{Z^{0}}$

The main decay channels in this region are $h^{0} \rightarrow \gamma \gamma$ and $A^{0} \rightarrow h^{0} Z^{0}$. Thus the $\gamma \gamma Z^{0}$ and $q \bar{q} \gamma \gamma \gamma(\gamma)$ topologies are the relevant ones. The vertex $A^{0} h^{0} Z^{0}$ is proportional to $\cos \delta$ 
and thus the decay $A^{0} \rightarrow h^{0} Z^{0}$ is dominant only for $\sin ^{2} \delta<0.95$. However, for higher $\sin ^{2} \delta$ values, these mass combinations are excluded by the limits on $h^{0} Z^{0}$ production.

II. $M_{h^{0}} / 2<M_{A^{0}}<M_{h^{0}}+M_{Z^{0}}$

$h^{0} \rightarrow \gamma \gamma$ and $A^{0} \rightarrow b \bar{b}$ are the dominant decays in most of the parameter space. However, the decay $A^{0} \rightarrow b \bar{b}$ is suppressed as $\sin ^{2} \delta$ approaches 0 , and the $A^{0}$ becomes stable, and thus invisible, for $\sin ^{2} \delta<10^{-6}$. The exclusion in this region comes from the search for $h^{0} A^{0}$ production, the results of a $\gamma \gamma+E_{\text {mis }}$ analysis (described in [3]) being used. This allows all the mass region excluded by the combination of the $h^{0} Z^{0}$ and $b \bar{b} \gamma \gamma$ analyses for higher $\sin ^{2} \delta$ values also to be excluded for low $\sin ^{2} \delta$.

III. $2 M_{b}<M_{A^{0}}<M_{h^{0}} / 2$

The decay $h^{0} \rightarrow A^{0} A^{0}$, kinematically allowed, becomes dominant. The $A^{0}$ still decays to $b \bar{b}$, as long as $2 M_{b}<M_{A^{0}}$, and this gives rise to 6 -fermion final states. This decay is relevant also for non-fermiophobic 2 HDMs, and the results published by DELPHI in [23] are used. For very low $\sin ^{2} \delta$ there is a region of totally invisible final states for which the results on the invisible $Z^{0}$ width, obtained at LEP1, are used, as explained in [3].

The LEP1 results on the total $Z^{0}$ width are also used to cover the region where the two masses are below the reach of the present analysis [3].

These exclusions are valid for all the allowed parameter space in the case of potential A, but only for $M_{H^{+}}>500 \mathrm{GeV} / c^{2}$ or $\sin ^{2} \delta>0.1$ in the case of potential B. For lower values of both $M_{H^{+}}$and $\sin ^{2} \delta$, the $\operatorname{BR}\left(h^{0} \rightarrow \gamma \gamma\right)$ can vanish due to cancellation of the different loop contributions.

For the two potentials considered, the exclusions obtained for invisible $A^{0}$ (which are more restrictive than the ones for visible final states, in region III) apply only to small mass regions. This is because $\sin ^{2} \delta<10^{-6}$ implies $M_{h^{0}} \sim 0$ in potential A and a band of $M_{h^{0}} \sim M_{A^{0}}$ in potential B. Both mass bands are in principle outside region III but, since their precise width is not known, the region of exclusion for invisible $A^{0}$ is also shown.

\section{Conclusions}

Around $650 \mathrm{pb}^{-1}$ of LEP2 data collected by DELPHI, at centre-of-mass energies between 183 and $209 \mathrm{GeV}$, were analysed in the search for Higgs bosons decaying into photons. In the context of $2 \mathrm{HDM}$, both $h^{0} Z^{0}$ and $h^{0} A^{0}$ production were searched for, and a large variety of final states involving photons and fermions was considered. No evidence for new physics was found.

Lower limits were set on the mass of a particle with Higgs-like couplings to bosons and decaying to two photons. In a model where the Higgs couplings to bosons have SM values, but the couplings to fermions vanish, a 95\% CL lower limit on the $h^{0}$ boson mass is set at $104.1 \mathrm{GeV} / c^{2}$; the expected limit in this case is $104.6 \mathrm{GeV} / c^{2}$. Exclusions at $95 \% \mathrm{CL}$ were also derived in the mass plane $\left(M_{h^{0}}, M_{A^{0}}\right)$ of the fermiophobic $2 \mathrm{HDM}$ scenario.

\section{Acknowledgements}

We are greatly indebted to our technical collaborators, to the members of the CERNSL Division for the excellent performance of the LEP collider, and to the funding agencies for their support in building and operating the DELPHI detector. 
We acknowledge in particular the support of Austrian Federal Ministry of Education, Science and Culture, GZ 616.364/2-III/2a/98, FNRS-FWO, Flanders Institute to encourage scientific and technological research in the industry (IWT), Belgium,

FINEP, CNPq, CAPES, FUJB and FAPERJ, Brazil,

Czech Ministry of Industry and Trade, GA CR 202/99/1362,

Commission of the European Communities (DG XII),

Direction des Sciences de la Matière, CEA, France,

Bundesministerium für Bildung, Wissenschaft, Forschung und Technologie, Germany,

General Secretariat for Research and Technology, Greece,

National Science Foundation (NWO) and Foundation for Research on Matter (FOM),

The Netherlands,

Norwegian Research Council,

State Committee for Scientific Research, Poland, SPUB-M/CERN/PO3/DZ296/2000, SPUB-M/CERN/PO3/DZ297/2000, 2P03B 10419 and 2P03B 69 23(2002-2004)

FCT - Fundação para a Ciência e Tecnologia, Portugal,

Vedecka grantova agentura MS SR, Slovakia, Nr. 95/5195/134,

Ministry of Science and Technology of the Republic of Slovenia,

CICYT, Spain, AEN99-0950 and AEN99-0761,

The Swedish Natural Science Research Council,

Particle Physics and Astronomy Research Council, UK,

Department of Energy, USA, DE-FG02-01ER41155.

EEC RTN contract HPRN-CT-00292-2002.

\section{References}

[1] K. Hagiwara, R. Szalapski and D. Zeppenfeld, Phys. Lett. B318 (1993) 155;

K. Hagiwara et al., Phys. Rev. D48 (1993) 2182.

[2] A. Barroso, L. Brücher, R. Santos, Phys. Rev. D60 (1999) 035005;

L. Brücher, R. Santos, Eur. Phys. J. C12 (2000) 87.

[3] DELPHI Coll., P. Abreu et al., Phys. Lett. B507 (2001) 89.

[4] DELPHI Coll., P. Abreu et al., Phys. Lett. B458 (1999) 431.

[5] ALEPH Coll., R. Barate et al., Phys. Lett. B487 (2000) 241;

L3 Coll., P. Achard et al., Phys. Lett. B534 (2002) 28;

OPAL Coll., G. Abbiendi et al., Phys. Lett. B544 (2002) 44.

[6] DELPHI Coll., P. Aarnio et al., Nucl. Instr. and Meth. A303 (1991) 233.

[7] DELPHI Coll., P. Abreu et al., Nucl. Instr. and Meth. A378 (1996) 57.

[8] DELPHI Silicon Tracker group, P. Chochula et al., Nucl. Instr. and Meth. A412 (1998) 304.

[9] S. Jadach, B.F.L. Ward and Z.Wạs, Comp. Phys. Comm. 130 (2000) 260.

[10] S. Jadach, B.F.L. Ward and Z.Wạs, Comp. Phys. Comm. 79 (1994) 503.

[11] S. Jadach, W. Placzek and B.F.L. Ward, Phys. Lett. B390 (1997) 298.

[12] T. Sjöstrand et al., Comp. Phys. Comm. 135 (2001) 238.

[13] A. Ballestrero et al., Comp. Phys. Comm. 152 (2003) 175.

[14] E. Accomando and A. Ballestrero, Comp. Phys. Comm. 99 (1997) 270;

E. Accomando, A. Ballestrero and E. Maina, Comp. Phys. Comm. 150 (2003) 166.

[15] F.A. Berends, P.H. Daverveldt and R. Kleiss, Comp. Phys. Comm. 40 (1986) 285;

F.A. Berends, P.H. Daverveldt and R. Kleiss, Comp. Phys. Comm. 40 (1986) 271. 
[16] F.A. Berends and R. Kleiss, Nucl. Phys. B186 (1981) 22.

[17] D. Karlen, Nucl. Phys. B289 (1987) 23.

[18] P. Janot "HZHA", in "Physics at LEP2", ed. G. Altarelli, T. Sjöstrand and F. Zwirner, CERN Report 1996-001 (1996) Vol. 2, 309.

[19] S. Catani et al., Phys. Lett. B269 (1991) 432.

[20] DELPHI Coll., P. Abreu et al., Eur. Phys. J. C2 (1998) 581.

[21] A.L. Read, "Modified Frequentist Analysis of Search Results (The $\mathrm{CL}_{s}$ Method)" in "Workshop on Confidence Limits", ed. F. James, L. Lyons and Y. Perrin, CERN Report 2000-005 (2000) p.81.

[22] DELPHI Coll., J. Abdallah et al., "b-tagging in DELPHI at LEP", CERN-EP/2002088, accepted by Eur. Phys. J. C.

[23] DELPHI Coll., J. Abdallah et al., "Searches for Neutral Higgs Bosons in Extended Models", CERN-EP/2003-061, submitted to Eur. Phys. J. C. 


\begin{tabular}{|c|r|r|r|r|r|}
\hline Selection & Data & Background & $q \bar{q}$ & eff $_{50}$ & eff $_{100}$ \\
\hline 2 photons & 338 & $341.5 \pm 9.5$ & $296.5 \pm 9.2$ & $60 \%$ & $60 \%$ \\
\hline no-ISR/FSR & 23 & $22.9 \pm 0.3$ & $21.2 \pm 0.3$ & $39 \%$ & $45 \%$ \\
\hline Z fit & 12 & $12.4 \pm 0.2$ & $12.3 \pm 0.2$ & $31 \%$ & $41 \%$ \\
\hline$\Delta E$ & 9 & $8.7 \pm 0.2$ & $8.6 \pm 0.2$ & $26 \%$ & $40 \%$ \\
\hline HPC & 9 & $8.4 \pm 0.2$ & $8.3 \pm 0.2$ & $26 \%$ & $40 \%$ \\
\hline
\end{tabular}

Table $2: h^{0} Z^{0} \rightarrow \gamma \gamma q \bar{q}$ selection in the year 2000: the evolution of the data sample with the analysis cuts is shown for the full 2000 data set, compared to the total expected background (and the $q \bar{q}$ background) and the efficiencies for Higgs masses of $50 \mathrm{GeV} / c^{2}$ and $100 \mathrm{GeV} / c^{2}$. The errors on the backgrounds are statistical only. The absolute statistical error on the efficiencies is $1-2 \%$.

\begin{tabular}{|c|r|r|r|c|}
\hline Selection & Data & Background & eff $_{50}$ & eff $_{100}$ \\
\hline 2 photons & 24 & $28.8 \pm 1.1$ & $36 \%$ & $36 \%$ \\
\hline no-ISR/FSR & 5 & $5.3 \pm 0.5$ & $22 \%$ & $31 \%$ \\
\hline E-p fit $+M_{Z^{0}}$ & 0 & $1.6 \pm 0.2$ & $17 \%$ & $27 \%$ \\
\hline
\end{tabular}

Table 3: $h^{0} Z^{0} \rightarrow \gamma \gamma l^{+} l^{-}$selection in the year 2000: the evolution of the data sample with the analysis cuts is shown together with the total expected background (with the corresponding statistical errors) and the efficiencies for Higgs masses of $50 \mathrm{GeV} / c^{2}$ and $100 \mathrm{GeV} / c^{2}$ (with $1-2 \%$ statistical error).

\begin{tabular}{|c|r|r|r|r|r|r|}
\hline Selection & Data & Background & QED & $\nu \bar{\nu} \gamma \gamma$ & eff $_{50}$ & eff $_{100}$ \\
\hline preselection & 1077 & $1121.2 \pm 7.9$ & $1110.6 \pm 7.9$ & $10.6 \pm 0.8$ & $60 \%$ & $64 \%$ \\
\hline $2 \gamma s: \theta_{\gamma} \notin\left[35^{\circ}, 42^{\circ}\right]$ & 879 & $924.5 \pm 7.2$ & $915.7 \pm 7.1$ & $8.8 \pm 0.7$ & $50 \%$ & $56 \%$ \\
\hline$\alpha_{\gamma \gamma}<178^{\circ}$ & 206 & $200.0 \pm 3.3$ & $191.2 \pm 3.2$ & $8.8 \pm 0.7$ & $50 \%$ & $56 \%$ \\
\hline QED veto & 13 & $9.0 \pm 0.7$ & $1.2 \pm 0.3$ & $7.8 \pm 0.7$ & $48 \%$ & $53 \%$ \\
\hline Z fit & 6 & $6.6 \pm 0.6$ & $0.5 \pm 0.2$ & $6.1 \pm 0.6$ & $44 \%$ & $50 \%$ \\
\hline
\end{tabular}

Table 4: $h^{0} Z^{0} \rightarrow \gamma \gamma \nu \bar{\nu}$ selection in the year 2000: the evolution of the data sample with the analysis cuts is shown, together with the total expected background (and the $Q E D$ and $\nu \bar{\nu} \gamma \gamma$ expectations) and corresponding statistical errors. The efficiencies for Higgs masses of $50 \mathrm{GeV} / c^{2}$ and $100 \mathrm{GeV} / c^{2}$ are shown in the last two columns, and have associated statistical errors of $1-2 \%$. 


\begin{tabular}{|c|c|c|c|c|c|c|}
\hline \multirow{2}{*}{$\begin{array}{l}\sqrt{s} \\
(\mathrm{GeV})\end{array}$} & \multicolumn{2}{|c|}{$\gamma \gamma q \bar{q}$} & \multicolumn{2}{|c|}{$\gamma \gamma l^{+} l^{-}$} & \multicolumn{2}{|c|}{$\gamma \gamma \nu \bar{\nu}$} \\
\hline & DATA & $\mathrm{MC}$ & DATA & $\mathrm{MC}$ & DATA & $\mathrm{MC}$ \\
\hline 183 & 4 & $2.64 \pm 0.12$ & - & - & $\overline{2}$ & $1.59 \pm 0.65$ \\
\hline 189 & 8 & $6.85 \pm 0.33$ & 1 & $1.70 \pm 0.44$ & 5 & $4.82 \pm 0.55$ \\
\hline 192 & 0 & $1.17 \pm 0.05$ & $\overline{0}$ & $0.27 \pm 0.07$ & 1 & $0.93 \pm 0.13$ \\
\hline 196 & 4 & $3.24 \pm 0.15$ & $\overline{2}$ & $0.62 \pm 0.15$ & $\overline{2}$ & $2.29 \pm 0.26$ \\
\hline 200 & 4 & $3.15 \pm 0.15$ & 0 & $0.60 \pm 0.15$ & 2 & $2.31 \pm 0.24$ \\
\hline 202 & 3 & $1.66 \pm 0.08$ & 0 & $0.25 \pm 0.07$ & 1 & $0.99 \pm 0.15$ \\
\hline 205 & 3 & $3.13 \pm 0.10$ & $\overline{0}$ & $0.61 \pm 0.16$ & 2 & $2.40 \pm 0.29$ \\
\hline 206.5 & 3 & $2.20 \pm 0.10$ & 0 & $0.29 \pm 0.07$ & 4 & $1.58 \pm 0.22$ \\
\hline 207 & 3 & $3.11 \pm 0.14$ & 0 & $0.69 \pm 0.17$ & 0 & $2.58 \pm 0.31$ \\
\hline TOT & 32 & $27.15 \pm 0.47$ & 3 & $5.03 \pm 0.55$ & 19 & $19.49 \pm 1.06$ \\
\hline
\end{tabular}

Table 5: Number of selected events for $h^{0} Z^{0}$ production and corresponding background expectations for the three topologies and all data samples considered. The errors on the background are statistical only.

\begin{tabular}{|c|r|r|r|r|}
\hline Selection & Data & Background & $q \bar{q}$ & eff $_{100}$ \\
\hline no-ISR/FSR & 23 & $22.9 \pm 0.3$ & $21.2 \pm 0.3$ & $53 \%$ \\
\hline fit & 19 & $20.1 \pm 0.3$ & $19.1 \pm 0.3$ & $49 \%$ \\
\hline$\Delta E$ & 14 & $17.1 \pm 0.3$ & $16.1 \pm 0.2$ & $49 \%$ \\
\hline HPC & 14 & $16.5 \pm 0.3$ & $15.5 \pm 0.3$ & $49 \%$ \\
\hline b-tag & 7 & $6.9 \pm 0.2$ & $6.4 \pm 0.2$ & $46 \%$ \\
\hline
\end{tabular}

Table 6: $h^{0} A^{0} \rightarrow \gamma \gamma b \bar{b}$ analysis: the comparison of data and MC events selected at each analysis level for the year 2000 data. The $q \bar{q}(\gamma \gamma)$ contribution is shown separately. The efficiency for the selection of a $h^{0} A^{0}$ signal with $M_{h^{0}}=100 \mathrm{GeV} / c^{2}$ and $M_{A^{0}}=M_{Z^{0}}$ produced at $\sqrt{s}=206 \mathrm{GeV}$ is shown in the last column (the corresponding statistical error being of $1-2 \%$ ).

\begin{tabular}{|c|r|r|r|r|}
\hline Selection & Data & Background & $q \bar{q}$ & eff \\
\hline no-ISR/FSR & 6 & $4.22 \pm 0.14$ & $4.00 \pm 0.14$ & $66 \%$ \\
\hline$Z^{0}$ mass & 3 & $3.31 \pm 0.13$ & $3.07 \pm 0.12$ & $66 \%$ \\
\hline$\Delta E$ & 2 & $1.85 \pm 0.10$ & $1.65 \pm 0.08$ & $64 \%$ \\
\hline HPC & 1 & $0.86 \pm 0.07$ & $0.73 \pm 0.06$ & $62 \%$ \\
\hline
\end{tabular}

Table 7: $h^{0} A^{0} \rightarrow q \bar{q} \gamma \gamma \gamma(\gamma)$ analysis: the comparison of selected data and MC at each analysis level for the year 2000 data is shown. The efficiency for the selection of a $h^{0} A^{0}$ signal with $M_{h^{0}}=35 \mathrm{GeV} / c^{2}$ and $M_{A^{0}}=135 \mathrm{GeV} / c^{2}$ produced at $\sqrt{s}=206 \mathrm{GeV}$ is shown in the last column (the corresponding statistical error being of 1-2\%). 


\begin{tabular}{|l|rr|rr|}
\hline$\sqrt{s}$ & \multicolumn{2}{|c|}{$b b \gamma \gamma$} & \multicolumn{2}{|c|}{$q \bar{q} \gamma \gamma \gamma(\gamma)$} \\
$(\mathrm{GeV})$ & DATA & MC & DATA & MC \\
\hline 183 & 2 & $1.90 \pm 0.10$ & 0 & $0.23 \pm 0.04$ \\
\hline 189 & 3 & $5.82 \pm 0.30$ & 0 & $0.74 \pm 0.11$ \\
\hline 192 & 1 & $0.94 \pm 0.05$ & 0 & $0.13 \pm 0.02$ \\
\hline 196 & 4 & $2.82 \pm 0.14$ & 1 & $0.29 \pm 0.05$ \\
\hline 200 & 3 & $2.91 \pm 0.15$ & 1 & $0.35 \pm 0.05$ \\
\hline 202 & 1 & $1.50 \pm 0.07$ & 0 & $0.16 \pm 0.02$ \\
\hline 205 & 6 & $2.43 \pm 0.09$ & 1 & $0.32 \pm 0.04$ \\
\hline 206.5 & 1 & $1.85 \pm 0.09$ & 0 & $0.20 \pm 0.03$ \\
\hline 207 & 0 & $2.59 \pm 0.10$ & 0 & $0.34 \pm 0.05$ \\
\hline TOT & 21 & $22.76 \pm 0.42$ & 3 & $2.75 \pm 0.15$ \\
\hline
\end{tabular}

Table 8: Selected events for $h^{0} A^{0}$ production and corresponding background expectations for the two topologies and all data samples considered. The errors on the background are statistical only. 


\section{DELPHI}
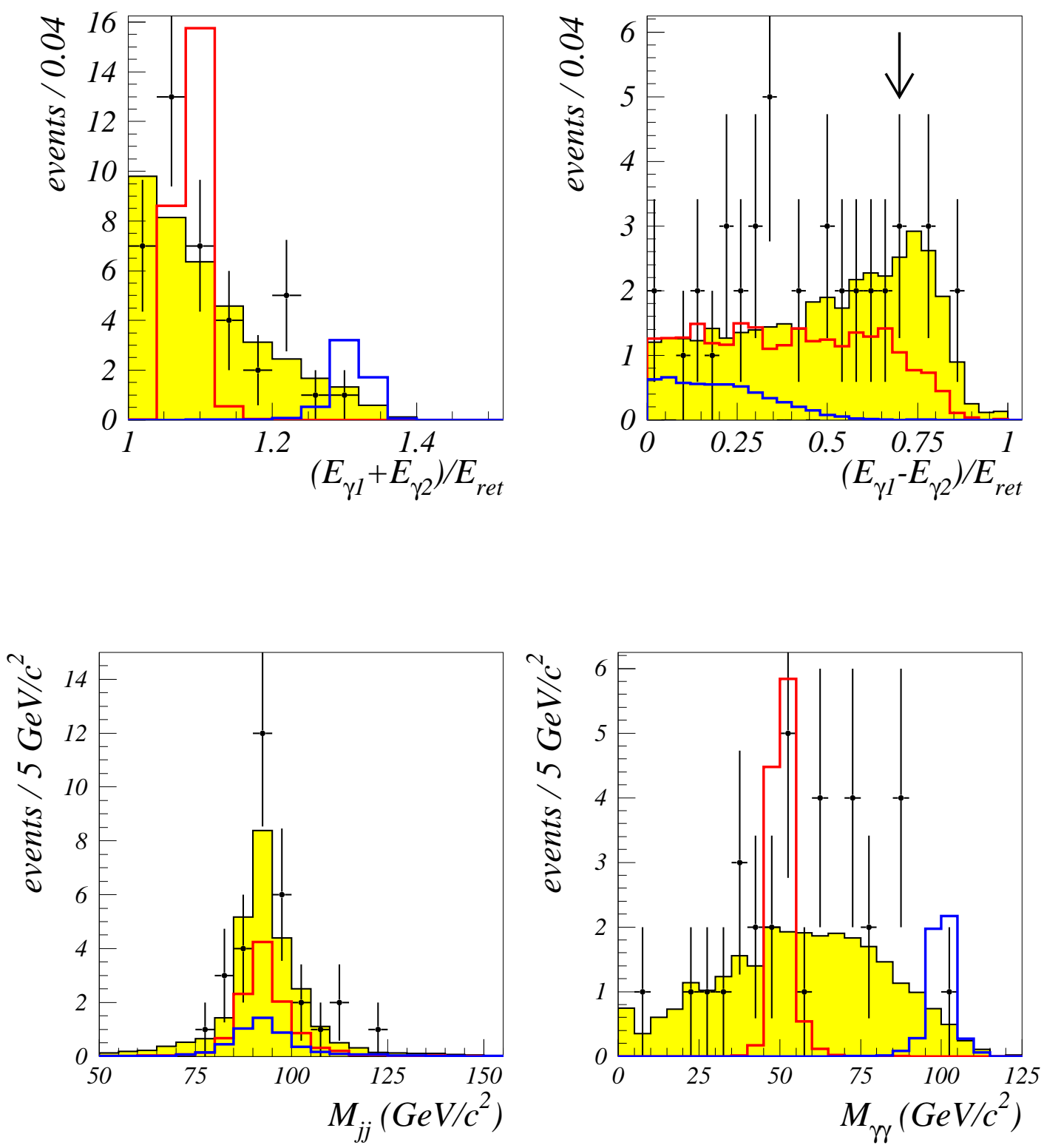

Figure 1: $h^{0} Z^{0} \rightarrow \gamma \gamma q \bar{q}$. The top plots show the sum (left) and difference (right) of the two photon energies divided by the radiative return energy before the cut on the energy difference (indicated by the arrow). The bottom plots show the reconstructed invariant jet-jet mass (left) and the fitted Higgs mass (right). The full data set (dots) is compared to the SM background (shaded area) and two $h^{0} Z^{0}$ signals: with $M_{h^{0}}=50 \mathrm{GeV} / c^{2}$ and $M_{h^{0}}=100 \mathrm{GeV} / c^{2}$ (thick lines), shown with arbitrary normalization. 


\section{DELPHI}
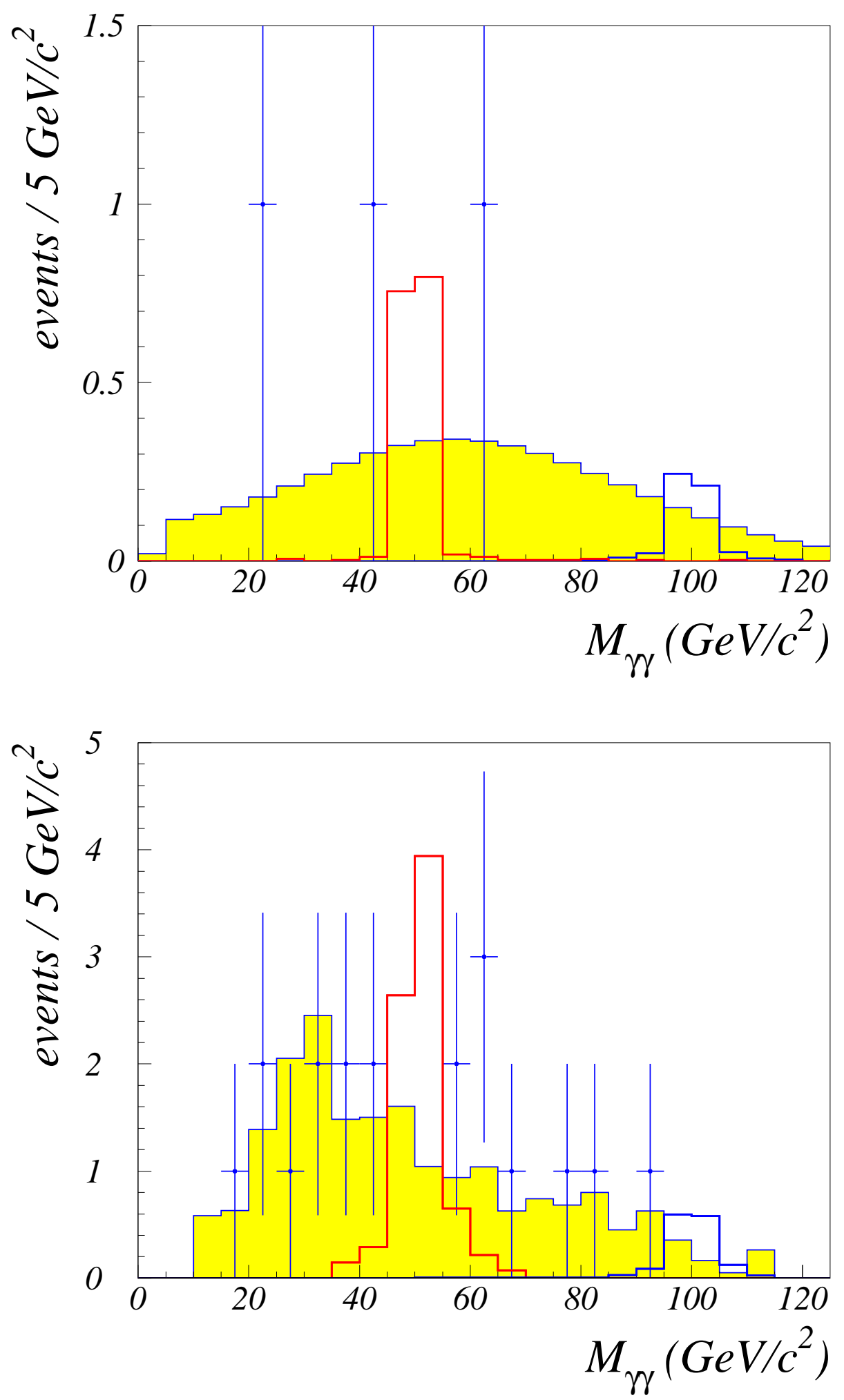

Figure 2: $h^{0} Z^{0} \rightarrow \gamma \gamma l^{+} l^{-}$(top) and $h^{0} Z^{0} \rightarrow \gamma \gamma \nu \bar{\nu}$ (bottom). The invariant masses $M_{\gamma \gamma}$ at the last selection level for all analysed data (dots) are compared to the SM background expectations (shaded histogram), and $h^{0} Z^{0}$ signals of 50 and $100 \mathrm{GeV} / c^{2}$ (top), shown with arbitrary normalization. 


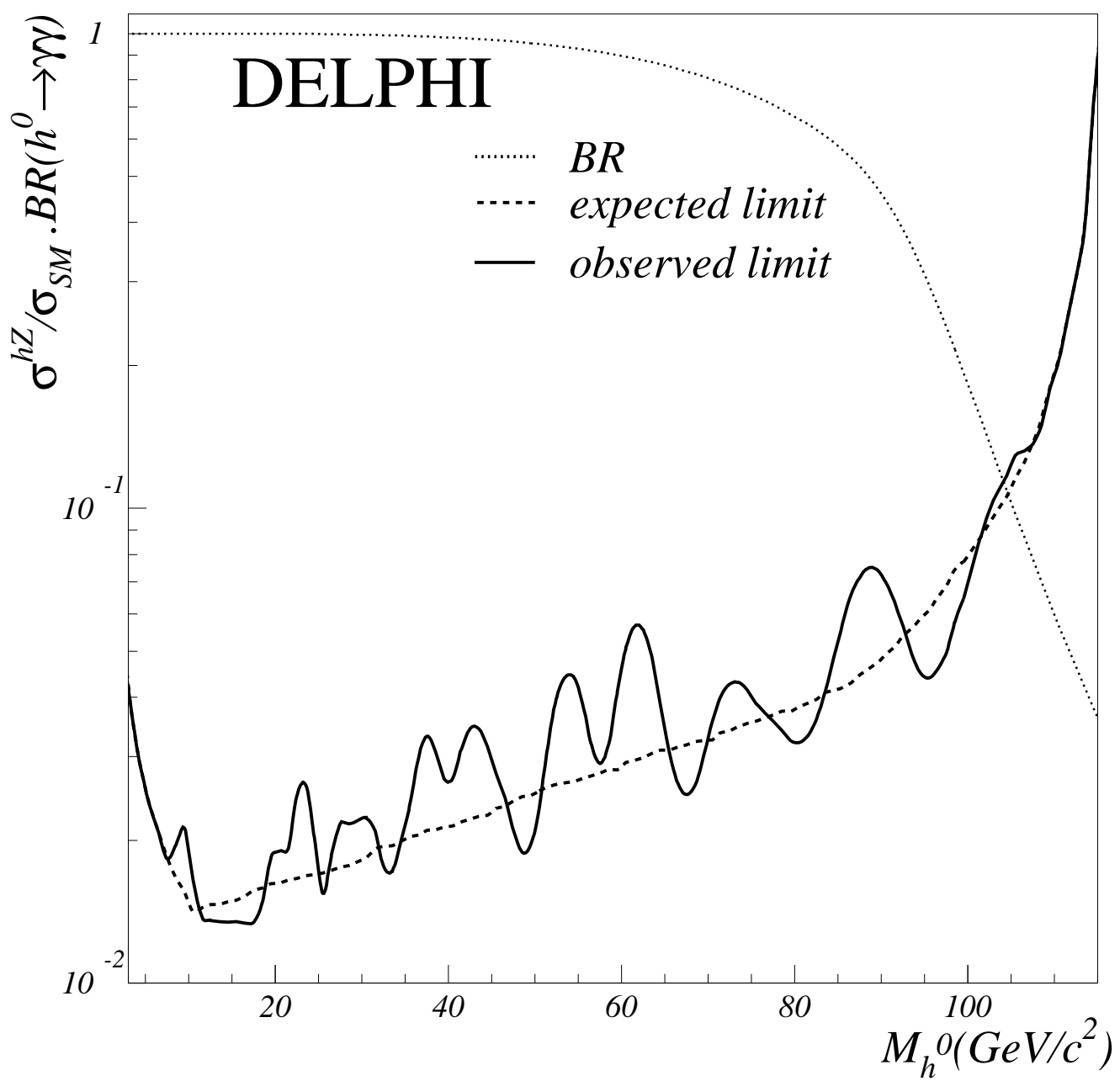

Figure 3: $95 \%$ CL upper limit on the $h^{0} Z^{0}$ production cross-section $\times \operatorname{BR}\left(h^{0} \rightarrow \gamma \gamma\right)$ normalized to the SM value. Both the observed (full line) and the expected limits (dashed line) are shown. Also shown is the fermiophobic $B R\left(h^{0} \rightarrow \gamma \gamma\right)$ (dotted line), obtained by keeping the SM couplings of the Higgs to boson pairs and setting the $h^{0} f \bar{f}$ couplings to 0 . A $95 \%$ CL mass limit is given by the intersection of the cross-section limit and the $B R\left(h^{0} \rightarrow \gamma \gamma\right)$ curve at $104.1 \mathrm{GeV} / c^{2}\left(104.6 \mathrm{GeV} / c^{2}\right.$ expected). 


\section{DELPHI}
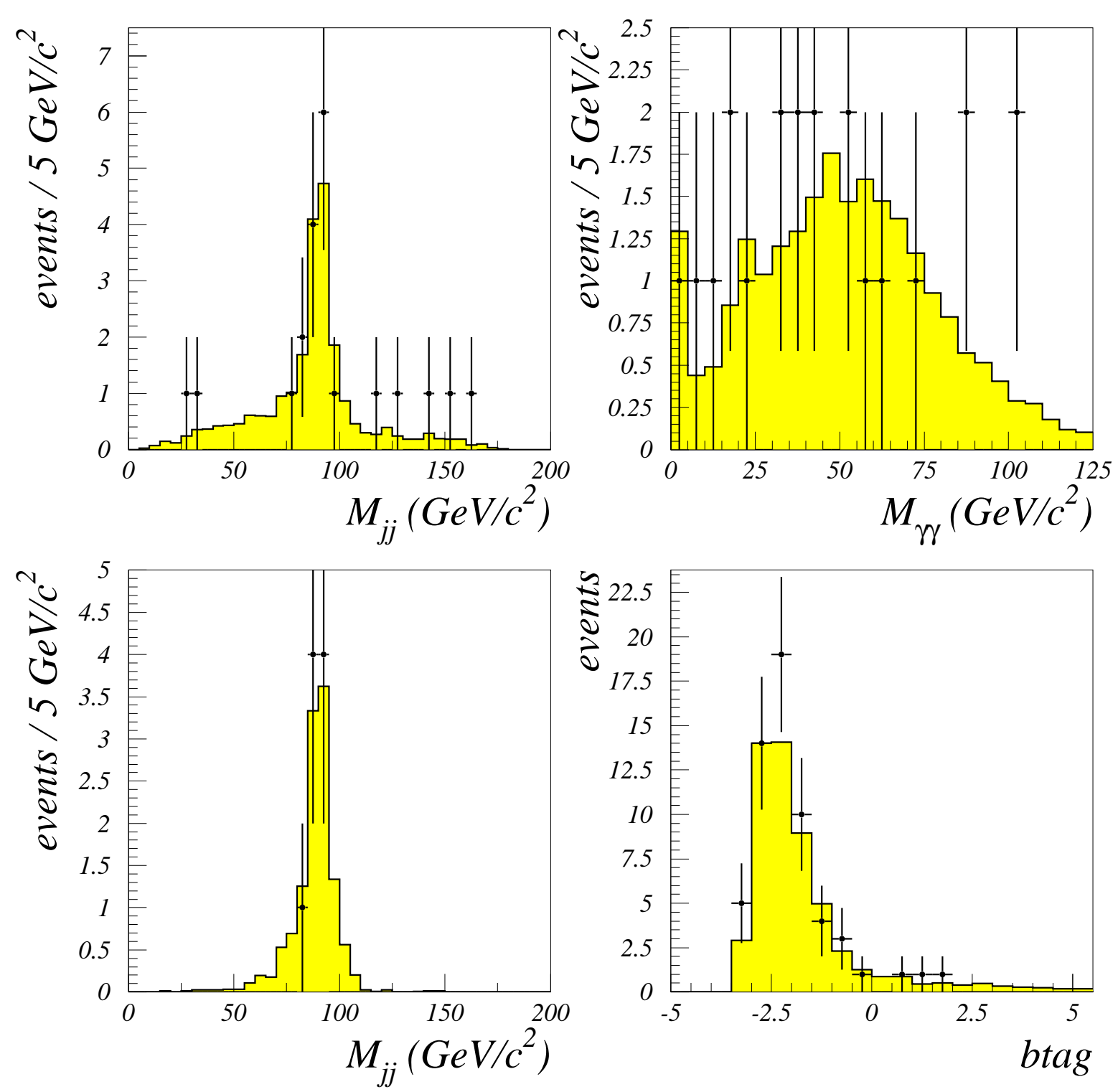

Figure 4: $h^{0} A^{0} \rightarrow \gamma \gamma b \bar{b}$ analysis. The reconstructed $A^{0}$ and $h^{0}$ masses at the final selection level are shown in the top plots for data and expected SM background in all data sets. The lower plots show the fitted jet-jet mass for events selected in both the $h^{0} A^{0}$ and $h^{0} Z^{0}$ analyses and the distribution of the b-tag variable just before the cut at -2 . 


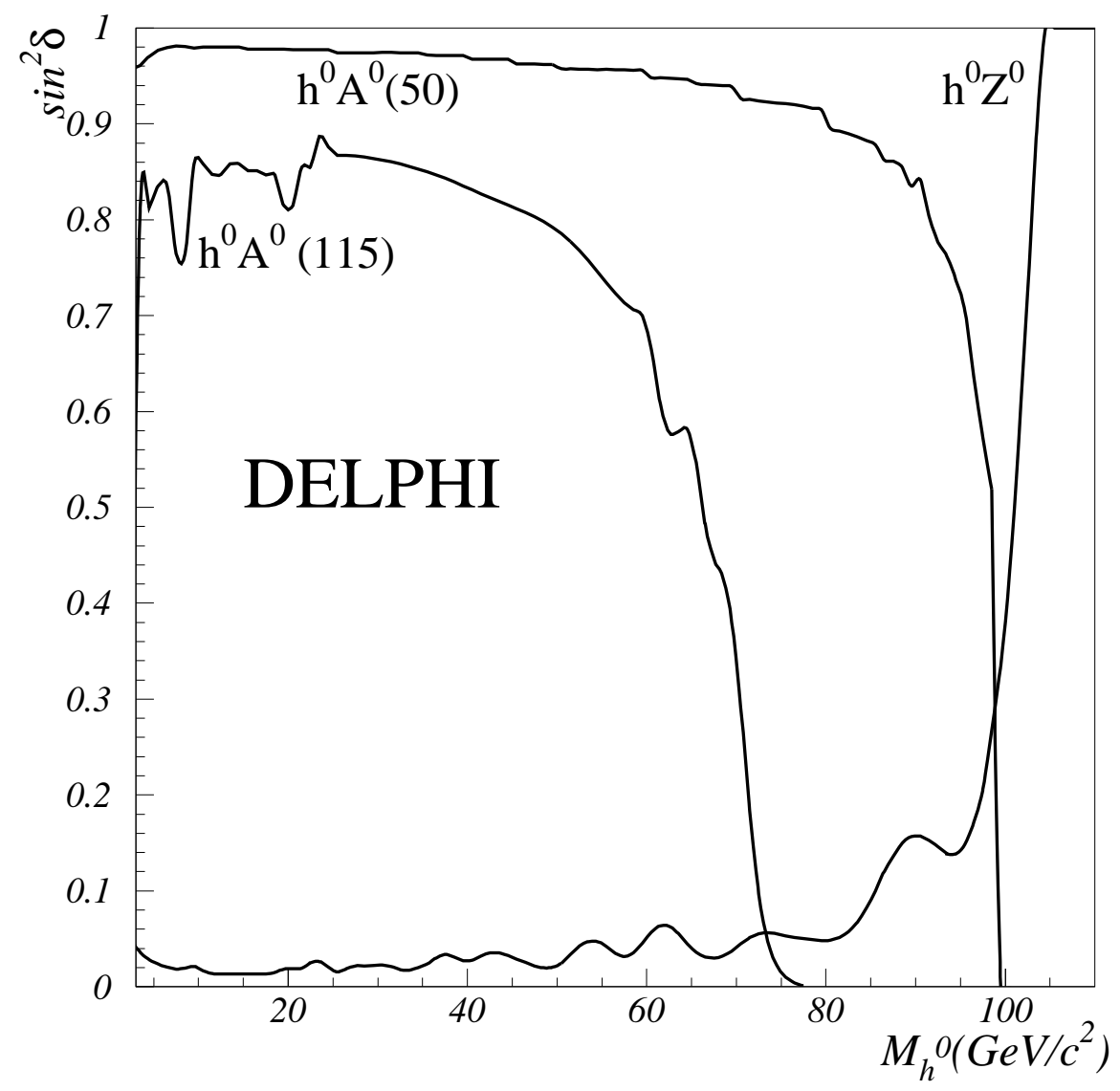

Figure 5: 95\% CL limits on the $h^{0} Z^{0}$ and $h^{0} A^{0}$ production cross-sections expressed in terms of $\sin ^{2} \delta$. Values above the $h^{0} Z^{0}$ curve and below the $h^{0} A^{0}$ curves (shown for two different $M_{A^{0}}$ values: $50 \mathrm{GeV} / c^{2}$ and $115 \mathrm{GeV} / c^{2}$ ) are excluded. 


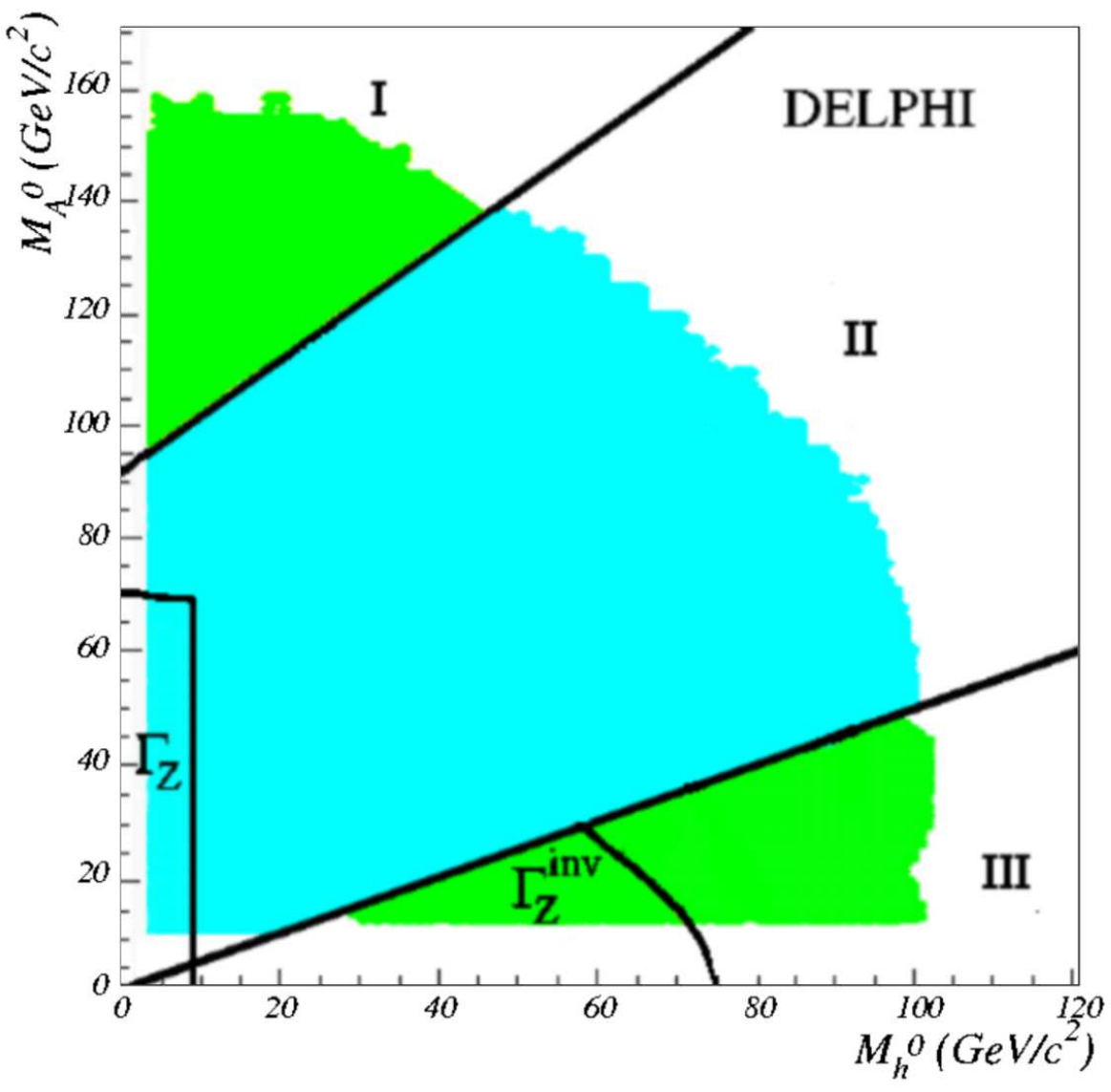

Figure 6: The shaded areas correspond to regions excluded at 95\% CL for all $\delta$ values. The plot is divided into regions according to the dominant decay modes of $h^{0}$ and $A^{0}$, as explained in the text. The exclusions from LEP 1 data, based on the total and invisible width of the $Z^{0}$, are also shown. 